\title{
Endoscopic third ventriculostomy in adult patients
}

\author{
Amin Amini, M.D., M.Sc., And Richard H. Schmidt, M.D., Ph.D. \\ Department of Neurosurgery, University of Utah School of Medicine, Salt Lake City, Utah
}

\begin{abstract}
Endoscopic third ventriculostomy (ETV) has gained popularity and has become the treatment of choice for certain pediatric and adult hydrocephalic conditions. The authors report their experience with 36 adult patients and evaluate the long-term outcome and safety of ETV. They discuss several improvements to the surgical techniques that they have developed based on their experience, including the use of intraoperative Doppler imaging before fenestration to trace the location of vessels underlying the floor of the third ventricle. They also report the use of a Rickham reservoir and endoventricular stent in selected cases and discuss the indications for their use. In cases of obstructive hydrocephalus due to congenital or acquired aqueductal stenosis in adults, the success rate of ETV in avoidance of shunt placement is $72 \%$. Twenty-two percent of the patients in this series in whom ETV was initially successful later experienced closure of the fenestration and recurrent symptoms at a mean interval of 3.75 years. Thus, in patients who undergo this treatment, long-term periodic follow-up review should be performed.
\end{abstract}

\section{KEY WORDS - endoscopic third ventriculostomy - adult chronic hydrocephalus • microvascular Doppler ultrasonography $\bullet$ stent}

\section{OVERVIEW}

Prior to the modern age of shunts, third ventriculostomy through an open craniotomy was the main treatment modality for hydrocephalus. As detailed in Hellwig, et al., ${ }^{21}$ Mixter first reported performing third ventriculostomy in 92 patients with hydrocephalus. Early high mortality and morbidity rates and the subsequent development of shunts decreased the use of third ventriculostomy. As the limitations of shunts for chronic hydrocephalus became more obvious, third ventriculostomy resurfaced as a plausible treatment for this condition. In 1973, Guiot ${ }^{20}$ described 20 patients with hydrocephalus in whom he had performed camera-guided third ventriculostomy, with a $75 \%$ success rate in control of the hydrocephalus. Today, ETV is a common practice in tertiary care neurosurgical facilities, and the list of indications for this procedure has grown.

Many factors have contributed to the attractiveness and popularity of ETV. Foremost is the fact that this procedure, when successful, eliminates reliance on mechanical shunts with all their limitations, and returns CSF dynamics to essentially normal status in patients with obstructive hydrocephalus. In addition, the simplicity and elegance of ETV and the measurable good outcome and high success rate among the appropriate patient population contribute to its wide popularity.

Abbreviations used in this paper: $\mathrm{AS}=$ aqueductal stenosis; $\mathrm{BA}=$ basilar artery; CSF = cerebrospinal fluid; ETV = endoscopic third ventriculostomy; ICP = intracranial pressure.
Initially, ETV was used as an aqueductal bypass procedure for the treatment of obstructive hydrocephalus. Early indications for ETV included hydrocephalus due to congenital and acquired AS secondary to brainstem and tectal tumors. Although some believe that congenital AS should not be treated with ETV because of the insufficiently developed subarachnoid space, ${ }^{21,41}$ others have successfully treated congenital hydrocephalus with this procedure. ${ }^{18}$

Today, many patients with hydrocephalus may be considered candidates for ETV. Currently, the surgical indications for the procedure include all of the following: primary $\mathrm{AS}, 6,10,11,17,19,23,29$ isolated lateral ventricular hydrocephalus in combination with endoscopic septal fenestration, $2,26,27,34,37$ idiopathic stenosis of the foramina of Magendie and Lusch$\mathrm{ka}^{31,50}$ Dandy-Walker malformation, ${ }^{9,36}$ and hydrocephalus due to intraventricular hematoma in combination with aspiration of the hematoma. ${ }^{14}$ In addition, ETV may be performed for hydrocephalus caused by mass effect from intraventricular tumors, ${ }^{6,15,16,54}$ pineal and tectal tumors, ${ }^{7,39}$ posterior fossa tumors, ${ }^{42,44}$ suprasellar arachnoid cysts, ${ }^{42}$ pineal cysts, ${ }^{16,51}$ or quadrigeminal cistern cysts. ${ }^{25}$ This procedure has also been reported to yield some limited success in the treatment of postinfectious/posthemorrhagic hydrocephalus ${ }^{49}$ slit ventricle syndrome,, 50 and communicating and normal-pressure hydrocephalus. ${ }^{32,35}$

With the dramatic rise in indications for ETV, we are faced with new neurosurgical complications and even more numerous technical difficulties. In this review, we present our experience with a series of adult patients and further 
discuss the surgical technique, complications, and possible solutions to some of these problems.

\section{Surgical Technique}

The standard surgical technique for ETV has been well described $^{4,22,28,30,33,38,43,52,53}$ and will not be detailed step by step in this paper. Several refinements of surgical technique that we believe are valuable and lead to either improved outcome or improved safety have been introduced over time. We will focus our discussion on these refinements.

\section{Preoperative Evaluation}

It is important to note that this technique is appropriate only for patients who have sufficiently large ventricles in which to introduce the endoscope to navigate into the third ventricle. Generally, the width of the third ventricle should be approximately $5 \mathrm{~mm}$ or greater. If a patient has slit ventricles caused by overshunting of CSF, it will first be necessary to externalize the shunt to control drainage until an adequate working diameter of the ventricles is achieved. Although stereotactic guidance can be used as a surgical adjunct, it is typically not necessary and was not used for any of our adult cases.

We find it beneficial to perform ETV with patients placed in a semisitting position with the head flexed further forward so that the entry burr hole is positioned superiorly. This helps to minimize loss of CSF and air entrapment into the ventricles or the subdural space, which can be a cause of protracted postoperative headache, nausea, and vomiting. Loss of CSF may also be a risk factor for postoperative subdural hematoma. Closing the burr hole incision under a constant stream of saline infusion also helps to minimize any air entrapment.

\section{Trajectory and Access to the Ventricular System}

The optimal working trajectory into the third ventricle is usually achieved with an entry burr hole placed at or just anterior to the coronal suture $(2.5-3 \mathrm{~cm}$ lateral to the midline). In most cases, this will provide a direct trajectory from the entry site through the foramen of Monro into the third ventricle. Once entry into the ventricle has been obtained with a conventional ventriculostomy catheter, we prefer to replace the catheter with a peel-away sheath (Neuroview Introducer Sheath; Neuronavigational Corp., Costa Mesa, CA) with a diameter just slightly larger than the endoscope to provide a working porthole into the anatomy. This enables easy insertion of the endoscope into the ventricle without repeated trauma to cerebral cortex and ependymal vessels. It also allows for convenient egress of saline irrigation, eliminating any risk of overpressurizing the ventricle. If any significant hemorrhage occurs in the ventricle as a result of the procedure, the peel-away sheath also facilitates copious irrigation until the hemorrhage is cleared.

\section{Identifying the BA Complex}

We have previously described a technique of using a microvascular Doppler probe inserted through the endoscope to locate precisely by insonation the position of the $\mathrm{BA}$ complex below the floor of the third ventricle..$^{45}$ Injury to the BA complex or its perforating vessels has been reported in multiple cases and can result in catastrophic and often fatal hemorrhage or brainstem infarction. Thus, precise knowledge of the location of this artery is essential before performing fenestration of the ventricle, and the fenestration should always be made anterior to the BA complex. In the ideal situation, this artery complex lies at the posterior edge of the third ventricle floor at the level of the mammillary bodies.

In our series of 36 adult patients, however, we found that variations in the relationship of the BA complex to the third ventricular floor occur frequently, and anomalies were noted in $14(39 \%)$ of our patients. These anomalies included anterior translocation of the BA complex, so that it lies underneath the midportion of the third ventricular floor, and ectatic anterior and upward bowing of an enlarged BA trunk, in which the vessels lie at risk underneath the midportion of the third ventricular floor. If the floor of the ventricle is translucent, these vascular anomalies can be readily detected and a safe entry site determined. In our series of patients, however, 19 patients (53\%) had thickened and opaque third ventricular floors, which rendered the underlying vascular anatomy optically invisible. The combination of an aberrantly located BA and an opaque third ventricular floor occurred in 19\% of our patients, placing them at increased risk for vascular injury.

We have found that microvascular Doppler ultrasonography can quickly and easily trace the exact position and layout of the underlying vessels when they are not optically visible, and this should help to minimize the risk of vascular injury. No cases of vascular injury occurred in our series. Learning the technique of microvascular Doppler examination of the floor of the third ventricle is facilitated in cases in which the vascular anatomy is visible (47\% of the patients in our series) and thus increases operator confidence in cases in which the ventricular floor is opaque (53\% of the patients in our series).

\section{Performing Ventriculostomy}

Because of the risk of vascular injury, the third ventricular floor should always be penetrated bluntly and never with laser energy or electrocautery. We prefer to use a $1-\mathrm{mm}$ blunt glass fiber to make the penetration; this is accomplished using a slight back-and-forth twisting motion. The initial fenestration is then dilated up to approximately a 4-mm opening by using a No. 2 French Fogarty catheter. In some cases, an imperforate membrane of Liliequist can be identified lying beneath the floor of the third ventricle, which would obstruct CSF outflow into the prepontine cistern. If such a membrane is present, it can be opened under direct endoscopic visualization with the glass fiber or balloon catheter.

\section{General Considerations and Closure}

We prefer to perform the endoscopic procedure with a steady flow of saline irrigation through the endoscope, using a gravity feed as opposed to pressure feed to avoid any barotrauma to the brain or ventricles. If any hemorrhaging is encountered during the procedure, copious warm saline irrigation should be used until all bleeding is visibly stopped and the ventricular CSF is clear. Once the endoscope and peel-away sheath are removed, we keep the ventricle inflated with a steady infusion of saline through a ventricular catheter while the burr hole incision is being closed. 
In recent years, it has become our custom in the majority of patients to leave a Rickham reservoir in place at the time of closure. As will be discussed later, this greatly facilitates management and diagnosis if patients are suspected to have persistent elevation of ICP because of deficient resorption of CSF or if the ventriculostomy site becomes closed over. In patients who are acutely ill at presentation with symptomatic elevated ICP, postoperative monitoring of the ICP and drainage of CSF can be accomplished through a needle ventriculostomy inserted percutaneously into the reservoir.

In patients in whom a Rickham reservoir has been left in place, we instill a combination of $10 \mathrm{mg}$ preservative-free vancomycin and $4 \mathrm{mg}$ preservative-free gentamicin into the ventricle at the time of closure. In a recent study, we have found that this regimen decreases the incidence of shunt infection 10-fold, to a rate of $0.5 \%$ (BT Ragel, et al., personal communication, 2005).

In two of our patients we found it expeditious to place a ventricular catheter stent through the third ventricular fenestration to maintain its patency (Fig. 1). In one of these patients, the stent was desirable because of early closing over of the ventricular fenestration caused by an ependymal cyst within the anterior half of the ventricle. In the second patient, the third ventricular floor was so stretched and patulous that, after fenestration, the redundant tissue tended to fold over on itself, causing concern for potential obstruction of the opening.

In both cases, stent placement was accomplished by inserting a ventricular catheter of appropriate length through the opening under endoscopic observation so that the distal catheter fenestrations lay within the prepontine cistern. A second set of fenestrations was created in the shaft of the ventricular catheter at the level of the lateral ventricle, and the catheter was secured in position with a Rickham reservoir. Long-term patency of the ventriculostomy has been achieved in both patients.

We prefer to keep our patients overnight in the neurosurgical intensive care unit for observation, and most patients can be discharged home the following day if they do not require postoperative ventricular drainage or ICP monitoring. If patients are doing well clinically, we defer postoperative imaging with computerized tomography or magnetic resonance imaging studies for 6 to 8 weeks to allow for stabilization of the ventricle size. After 6 to 8 weeks, an image is obtained for use as the baseline for subsequent follow-up evaluations. Even in those with clinically successful outcomes, and especially in adult patients with chronic compensated hydrocephalus, the ventricle size may decrease only minimally compared with its preoperative size..$^{8,47,48}$

\section{Outcome and Complications}

The incidence of complications with ETV has been reported ranging from 0 to $20 \%,,^{17,22,29,46}$ and the mortality rate has been reported as high as $1 \% .46,54$ The mortality rate appears to be directly related to the learning curve of the surgeon. ${ }^{46}$ The most devastating intraoperative complication is injury to the BA and its branches lying underneath the floor of the third ventricle. ${ }^{1,24}$ Common technical complications include bleeding at the level of the choroid plexus, ventricular wall, ventriculostomy, or interpeduncular cistern. Intraparenchymal or subdural bleeding and infection are less common complications of ETV. ${ }^{17}$

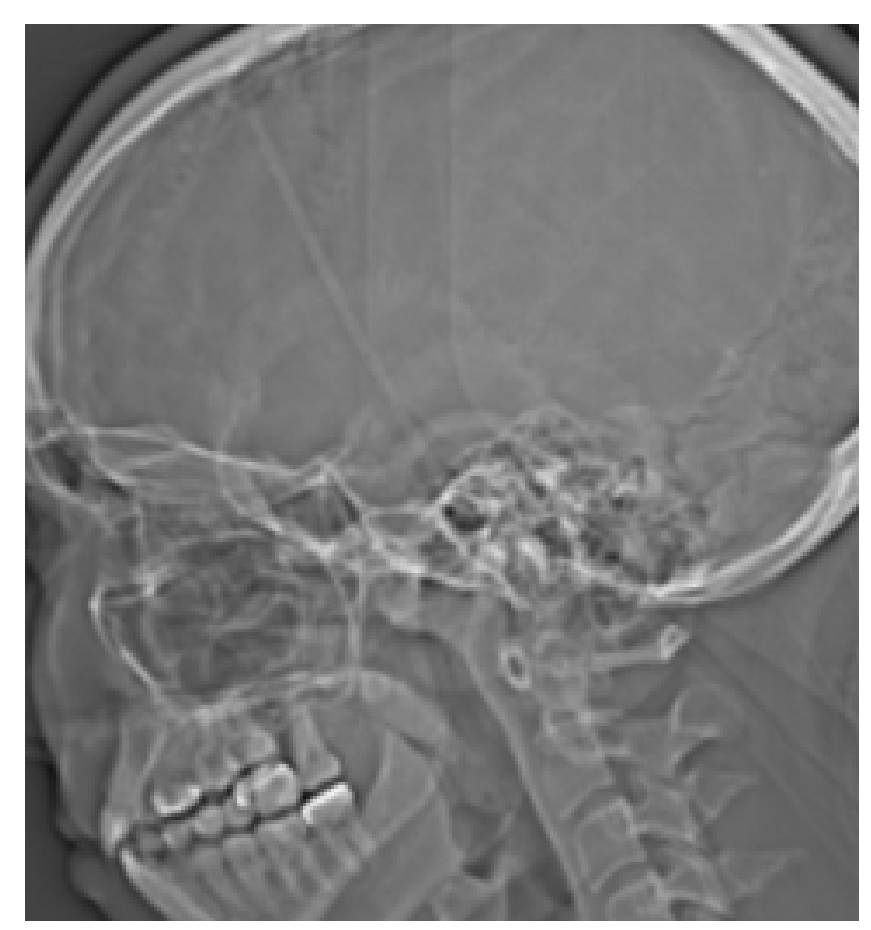

Fig. 1. Lateral X-ray film showing an intraventricular stent passing through the lateral ventricle, the foramen of Monro, and the floor of the third ventricle into the prepontine cistern. The proximal end is secured to a Rickham reservoir.

Data on our case series of 36 adult patients who underwent ETV for hydrocephalus are presented in Table 1. Our overall success rate in avoiding the need for a ventricular shunt was $61 \%$. In only one patient were we unable to perform ventricular fenestration because of unfavorable anatomy at the floor of the third ventricle. Ventricular fenestration was performed in all other patients, but $37 \%$ of them later required implantation of a ventricular shunt.

In our case series, $56 \%$ of the patients had congenital AS, and of this subgroup, $70 \%$ presented with adult onset of symptoms (chronic compensated hydrocephalus) and had not received a shunt previously. Seventy-two percent of these patients without shunts had a successful outcome after an ETV and did not require shunt placement. Of those patients who had a shunt in place, $57 \%$ became shunt free after ETV. Forty-three percent of patients presented with acquired obstructive hydrocephalus and $58 \%$ of these had AS secondary to a tectal plate glioma. Other causes included higher-grade tumors of the posterior fossa or pineal region, ventricular cysts, and one case of cysticercosis. Among this group of patients with acquired obstructive hydrocephalus, $58 \%$ overall were able to avoid shunt placement after ETV.

No surgically related deaths or major morbidity occurred in our case series. Complications included transient shortterm memory difficulty in one patient. There was one case of bacterial ventriculitis, and one patient suffered an intraventricular hemorrhage from a tumor biopsy procedure that was performed simultaneously with ventriculostomy. In one patient CSF leakage developed through the burr hole incision site because of persistently high ICP; she required treatment with a ventricular shunt. 
TABLE 1

Results in a series of 36 adult patients with hydrocephalus who underwent ETV

\begin{tabular}{lc}
\hline \multicolumn{1}{c}{ Factor } & No. of Patients $(\%)$ \\
\hline diagnosis & $20(56)$ \\
congenital AS & $12(33)$ \\
acquired AS & $3(8)$ \\
posterior fossa tumor & $1(3)$ \\
cysticercosis & \\
ventricular floor & $17(47)$ \\
translucent & $19(53)$ \\
opaque & $22(61)$ \\
vascular anatomy & $14(39)$ \\
normal & $8(22)$ \\
abnormal & \\
opaque \& abnormal & $22(61)$ \\
outcome & $14(39)$ \\
shunt free & \\
shunt dependent & $1(3)$ \\
complications & $1(3)$ \\
memory problems & $1(3)$ \\
unsuccessful ETV & $1(3)$ \\
infection & $1(3)$ \\
minimal hemorrhage & $5(14)$ \\
CSF leak & \\
overall & \\
\hline
\end{tabular}

The literature on ETV describes various treatment-related complications that we did not find in our case series. Such complications have included endocrinopathy caused by hypothalamic or pituitary stalk injury, which typically manifests as diabetes insipidus. ${ }^{12,18}$ Third and sixth cranial nerve palsies have been encountered and may be caused by direct nerve injury, barotrauma from overpressurization of the ventricles with irrigation fluid, or injury to one or more perforating arteries at the BA apex. Direct vascular injury to the BA apex or the proximal posterior cerebral artery has been described as a cause of either fatal hemorrhage or a traumatic aneurysm, but fortunately this complication is rare. ${ }^{1,24}$ Injury to the fornix can be caused by direct trauma from the endoscope while navigating through the foramen of Monro, and it typically leads to postoperative cognitive problems. Injury can also occur to the caudate, the thalamus, or the thalamostriate venous complex, especially if the endoscope is blindly overinserted into the ventricle. ${ }^{18}$ Cardiac arrhythmias or respiratory arrest have been encountered and typically result from manipulation or irritation of the hypothalamus. ${ }^{3,13}$ Subdural hemorrhage is a risk, especially in patients with very large ventricles who experience excessive CSF loss during the procedure. ${ }^{17,18}$ All of these potential complications should be taken into consideration when performing an ETV. The technical recommendations described in the preceding section have been developed to minimize the chance of these various complications occurring and appear to be effective; that is, we have avoided these problems.

\section{Repeated Closure and Operation}

Over the long term, three of our patients died of progression of malignant brain tumors. One patient with a tectal plate glioma died of recurrent hydrocephalus at a remote hospital 3 years after a successful third ventriculostomy. $\mathrm{Au}-$ topsy demonstrated closing over of the ventricular fenestration. Including this one, five of our 22 patients with initially successful ETVs experienced reclosure of their ventriculostomy. In one patient, as described previously, early obstruction by a third ventricular cyst developed, and the lesion was successfully treated with insertion of a stent. Four other patients presented again between 3 and 5 years after the initial operation, with a mean follow-up duration of 3.75 years. Except for the patient who died at another facility, these patients all underwent successful reoperation at our facility and remain shunt free.

In the patient with the longest follow-up duration (6 years), no reclosure has occurred. Because the overall incidence of ventriculostomy reclosure is relatively frequent $(22 \%)$, we recommend that all patients with initially successful ETVs be followed on a regular basis. Moreover, these patients, their families, and their physicians should be clearly warned about the possibility of recurrent hydrocephalus. We do not yet have enough experience with endoventricular stents to know if this is a potential long-term solution to the problem of ventriculostomy reclosure.

Determining whether reclosure of the fenestration and recurrent hydrocephalus has developed can be clinically difficult, especially in the adult population presenting with chronic compensated hydrocephalus. In these patients, a return of symptoms may be fairly subtle and slow in onset. Because the ventricle size may change only minimally in these patients, this is not always a reliable indicator. If obstructive hydrocephalus has recurred, a diagnostic lumbar puncture may not reflect pressure within the ventricles and could also be potentially unsafe. Cine-flow magnetic resonance imaging may be helpful to demonstrate CSF flow through the ventricular fenestration; however, technical difficulties with such studies may yield false-negative results. If the return of clinical symptoms is compelling and diagnostic studies yield equivocal results regarding the status of the ventriculostomy, endoscopic reexploration is recommended.

We have performed such endoscopic reexploration in two of our 22 patients and demonstrated a patent ventricular opening. Implanting a Rickham reservoir at the time of initial surgery greatly simplifies the problem of determining the status of a patient's hydrocephalus and the question of reclosure of the ventriculostomy. Assessment is done by percutaneous needle tap or, if necessary, by contrast ventriculogram. Moreover, the implanted reservoir can provide an avenue for emergency treatment if a patient presents with acute decompensation.

\section{Success Rate}

The overall shunt-free success rate of ETV has been reported to be between 50 and $90 \%$. Beems and Grotenhuis ${ }^{6}$ reported a complication rate of $7.7 \%$ and a success rate of $76 \%$ in a large series of 339 pediatric patients. In another series of adult and pediatric patients, Hopf, et al., ${ }^{23}$ reported that their success rate with ETV was $76 \%$ and concluded that ETV is most successful in occlusive hydrocephalus. Gangemi, et al., ${ }^{17}$ presented follow-up results in 125 adult and pediatric patients who underwent ETV for obstructive hydrocephalus. They reported overall shunt independence rates of $86.4 \%$. Most of the reported series involve either pediatric patients or a mixed population. Our series in- 
cludes adult patients exclusively and provides long-term follow-up results. Our success rate, which is defined by shunt independence, was $61 \%$, and this is comparable to previously reported outcomes. The overall complication rate was 14\%; none of these complications led to any permanent sequelae. As has been reported in other studies, we had a high success rate in patients with chronic compensated hydrocephalus and tectal plate glioma, suggesting that ETV should be the treatment of choice in these patients.

\section{CONCLUSIONS}

Our case series confirms that ETV is an effective method of treatment for adult patients with hydrocephalus, especially in those with chronic compensated hydrocephalus and brainstem tumors. To avoid some of the reported complications, we recommend regular use of a peel-away sheath and microvascular Doppler ultrasonography studies to avoid injury to the cerebral cortex, the ependymal vessels, and the BA complex. Although long-term follow-up results are very encouraging, patients with successful ETV remain at risk of reclosure of the fenestration, which can lead to a fatal outcome if not promptly recognized and treated. Therefore, we recommend that all patients with initially successful ETV receive follow-up care on a regular basis. Moreover, these patients, their families, and their physicians should be clearly warned about the possibility of recurrent hydrocephalus. Evaluation of patency of the ETV remains difficult. We have had encouraging experience with placement of a Rickham reservoir, but long-term follow-up review is needed to study its long-term efficacy. Although we have had successful results with stent placement, our experience is very limited and a firm conclusion is premature.

\section{Acknowledgment}

We thank Kristin Kraus for her expeditious editorial help with this paper.

\section{References}

1. Abtin K, Thompson BG, Walker ML: Basilar artery perforation as a complication of endoscopic third ventriculostomy. Pediatr Neurosurg 28:35-41, 1998

2. Aldana PR, Kestle JR, Brockmeyer DL, et al: Results of endoscopic septal fenestration in the treatment of isolated ventricular hydrocephalus. Pediatr Neurosurg 38:286-294, 2003

3. Anandh B, Madhusudan Reddy KR, Mohanty A, et al: Intraoperative bradycardia and postoperative hyperkalemia in patients undergoing endoscopic third ventriculostomy. Minim Invasive Neurosurg 45:154-157, 2002

4. Avman N, Kanpolat Y: Third ventriculostomy by microtechnique. Acta Neurochir Suppl (Wien) 28:588-595, 1979

5. Baskin JJ, Manwaring KH, Rekate HL: Ventricular shunt removal: the ultimate treatment of the slit ventricle syndrome. J Neurosurg 88:478-484, 1998

6. Beems T, Grotenhuis JA: Is the success rate of endoscopic third ventriculostomy age-dependent? An analysis of the results of endoscopic third ventriculostomy in young children. Childs Nerv Syst 18:605-608, 2002

7. Buxton N, Robertson I: Endoscopic approach to tectal tumors. J Neurosurg 93:152-153, 2000

8. Buxton N, Turner B, Ramli N, et al: Changes in third ventricular size with neuroendoscopic third ventriculostomy: a blinded study. J Neurol Neurosurg Psychiatry 72:385-387, 2002
9. Cartmill M, Vloeberghs M: The fate of the cerebrospinal fluid after neuroendoscopic third ventriculostomy. Childs Nerv Syst 16:879-881, 2000

10. Cinalli G, Sainte-Rose C, Chumas P, et al: Failure of third ventriculostomy in the treatment of aqueductal stenosis in children. J Neurosurg 90:448-454, 1999

11. Cinalli G, Sainte-Rose C, Simon I, et al: Sylvian aqueduct syndrome and global rostral midbrain dysfunction associated with shunt malfunction. J Neurosurg 90:227-236, 1999

12. Di Roio C, Mottolese C, Cayrel V, et al: Ventriculostomie du troième ventricule et diabète insipide. Ann Fr Anesth Reanim 18:776-778, 1999

13. Enya S, Masuda Y, Terui K: [Respiratory arrest after a ventriculoscopic surgery in infants: two case reports.] Masui 46: 416-420, 1997 (Jpn)

14. Fuentes S, Metellus P, Dufour H, et al: Apport de l'endoscopie dans la prise en charge des hématomes intra-ventriculaires supratentorials compliqués d'hydrocéphalie. À propos de deux cas. Neurochirurgie 48:510-515, 2002

15. Fukushima T: Endoscopic biopsy of intraventricular tumors with the use of a ventriculofiberscope. Neurosurgery 2:110-113, 1978

16. Gaab MR, Schroeder HW: Neuroendoscopic approach to intraventricular lesions. J Neurosurg 88:496-505, 1998

17. Gangemi M, Donati P, Maiuri F, et al: Endoscopic third ventriculostomy for hydrocephalus. Minim Invasive Neurosurg 42:128-132, 1999

18. Grant JA, McLone DG: Third ventriculostomy: a review. Surg Neurol 47:210-212, 1997

19. Grunert P, Charalampaki P, Hopf N, et al: The role of third ventriculostomy in the management of obstructive hydrocephalus. Minim Invasive Neurosurg 46:16-21, 2003

20. Guiot G: Ventriculo-cisternostomy for stenosis of the aqueduct of Sylvius. Puncture of the floor of the third ventricle with a leucotome under television control. Acta Neurochir (Wien) 28:275-289, 1973

21. Hellwig D, Grotenhuis JA, Tirakotai W, et al: Endoscopic third ventriculostomy for obstructive hydrocephalus. Neurosurg Rev 28:1-38, 2005

22. Hoffman HJ, Harwood-Nash D, Gilday DL: Percutaneous third ventriculostomy in the management of noncommunicating hydrocephalus. Neurosurgery 7:313-321, 1980

23. Hopf NJ, Grunert P, Fries G, et al: Endoscopic third ventriculostomy: outcome analysis of 100 consecutive procedures. Neurosurgery 44:795-806, 1999

24. Horowitz M, Albright AL, Jungreis C, et al: Endovascular management of a basilar artery false aneurysm secondary to endoscopic third ventriculostomy: case report. Neurosurgery 49: 1461-1465, 2001

25. Inamasu J, Ohira T, Nakamura Y, et al: Endoscopic ventriculocystomy for non-communicating hydrocephalus secondary to quadrigeminal cistern arachnoid cyst. Acta Neurol Scand 107: 67-71, 2003

26. Inamura T, Morioka $\mathrm{T}$, Nishio S, et al: Diverticular enlargement of the foramina of Luschka and congenital hydrocephalus. Childs Nerv Syst 18:652-655, 2002

27. Jabaudon D, Charest D, Porchet F: Pathogenesis and diagnostic pitfalls of ventricular diverticula: case report and review of the literature. Neurosurgery 52:209-212, 2003

28. Jaksche H, Loew F: Burr hole third ventriculo-cisternostomy. An unpopular but effective procedure for treatment of certain forms of occlusive hydrocephalus. Acta Neurochir (Wien) 79: 48-51, 1986

29. Jones RF, Kwok BC, Stening WA, et al: The current status of endoscopic third ventriculostomy in the management of noncommunicating hydrocephalus. Minim Invasive Neurosurg 37:28-36, 1994

30. Kamikawa S, Inui A, Tamaki N, et al: Application of flexible neuroendoscopes to intracerebroventricular arachnoid cysts in 
children: use of videoscopes. Minim Invasive Neurosurg 44: 186-189, 2001

31. Karachi C, Le Guerinel C, Brugieres P, et al: Hydrocephalus due to idiopathic stenosis of the foramina of Magendie and Luschka. Report of three cases. J Neurosurg 98:897-902, 2003

32. Kehler U, Gliemroth J: Extraventricular intracisternal obstructive hydrocephalus - a hypothesis to explain successful 3rd ventriculostomy in communicating hydrocephalus. Pediatr Neurosurg 38:98-101, 2003

33. Kunz U, Goldmann A, Bader C, et al: Endoscopic fenestration of the 3 rd ventricular floor in aqueductal stenosis. Minim Invasive Neurosurg 37:42-47, 1994

34. Lewis AI, Keiper GL Jr, Crone KR: Endoscopic treatment of loculated hydrocephalus. J Neurosurg 82:780-785, 1995

35. Meier U, Zeilinger FS, Schonherr B: Endoscopic ventriculostomy versus shunt operation in normal pressure hydrocephalus: diagnostics and indication. Minim Invasive Neurosurg 43: 87-90, 2000

36. Mohanty A: Endoscopic third ventriculostomy with cystoventricular stent placement in the management of Dandy-Walker malformation: technical case report of three patients. Neurosurgery 53:1223-1229, 2003

37. Oi S, Hidaka M, Honda Y, et al: Neuroendoscopic surgery for specific forms of hydrocephalus. Childs Nerv Syst 15:56-68, 1999

38. Oka K, Yamamoto M, Ikeda K, et al: Flexible endoneurosurgical therapy for aqueductal stenosis. Neurosurgery 33:236-243, 1993

39. Pople IK, Athanasiou TC, Sandeman DR, et al: The role of endoscopic biopsy and third ventriculostomy in the management of pineal region tumours. Br J Neurosurg 15:305-311, 2001

40. Reddy K, Fewer HD, West M, et al: Slit ventricle syndrome with aqueduct stenosis: third ventriculostomy as definitive treatment. Neurosurgery 23:756-759, 1988

41. Sainte-Rose C: Third ventriculostomy, in Manwaring KH, Crone KR (eds): Neuroendoscopy. New York: Mary Ann Liebert, 1992, pp 47-62

42. Sainte-Rose C, Cinalli G, Roux FE, et al: Management of hydrocephalus in pediatric patients with posterior fossa tumors: the role of endoscopic third ventriculostomy. J Neurosurg 95: 791-797, 2001

43. Sayers MP, Kosnik EJ: Percutaneous third ventriculostomy: experience and technique. Childs Brain 2:24-30, 1976
44. Schijman E, Peter JC, Rekate HL, et al: Management of hydrocephalus in posterior fossa tumors: how, what, when? Childs Nerv Syst 20:192-194, 2004

45. Schmidt RH: Use of a microvascular Doppler probe to avoid basilar artery injury during endoscopic third ventriculostomy. Technical note. J Neurosurg 90:156-159, 1999

46. Schroeder HW, Niendorf WR, Gaab MR: Complications of endoscopic third ventriculostomy. J Neurosurg 96:1032-1040, 2002

47. Schwartz TH, Ho B, Prestigiacomo CJ, et al: Ventricular volume following third ventriculostomy. J Neurosurg 91:20-25, 1999

48. Schwartz TH, Yoon SS, Cutruzzola FW, et al: Third ventriculostomy: post-operative ventricular size and outcome. Minim Invasive Neurosurg 39:122-129, 1996

49. Siomin V, Cinalli G, Grotenhuis A, et al: Endoscopic third ventriculostomy in patients with cerebrospinal fluid infection and/or hemorrhage. J Neurosurg 97:519-524, 2002

50. Suehiro T, Inamura T, Natori Y, et al: Successful neuroendoscopic third ventriculostomy for hydrocephalus and syringomyelia associated with fourth ventricle outlet obstruction. Case report. J Neurosurg 93:326-329, 2000

51. Tirakotai W, Riegel T, Schulte DM, et al: Neuroendoscopic stent procedure in obstructive hydrocephalus due to both foramina of monro occluding craniopharyngioma: technical note. Surg Neurol 61:293-296, 2004

52. Vries JK: An endoscopic technique for third ventriculostomy. Surg Neurol 9:165-168, 1978

53. Yamamoto M, Oka K, Ikeda K, et al: Percutaneous flexible neuroendoscopic ventriculostomy in patients with shunt malfunction as an alternative procedure to shunt revision. Surg Neurol 42:218-223, 1994

54. Yurtseven T, Ersahin Y, Demirtas E, et al: Neuroendoscopic biopsy for intraventricular tumors. Minim Invasive Neurosurg 46:293-299, 2003

Manuscript received October 15, 2005.

Accepted in final form November 11, 2005.

Address reprint requests to: Richard H. Schmidt, M.D., Ph.D., Department of Neurosurgery, University of Utah School of Medicine, 30 N 1900 E, Suite 3B409, Salt Lake City, Utah 84132. email: Richard.Schmidt@hsc.utah.edu. 